\title{
À quoi servent les graphiques
}

\author{
Sémir BADIR \\ Fonds National belge de la Recherche Scientifique \\ Université de Liège
}

J'avais pensé présenter dans cette étude les caractéristiques formelles d'un corpus d'images graphiques. En effet, ma première ambition était de travailler sur les graphiques qui ont pour objets des sons. La question qui avait motivé le choix de ce corpus particulier était celle de la polysensorialité (comment deux sens se rencontrent-ils ?), question qui entraîne avec elle la problématique de la polysémioticité (de quelle manière et dans quelle mesure les graphiques, relevant du domaine des énoncés visuels, s'y prennent-ils pour représenter des objets qui ne sont pas visuels ?). De ce corpus, je me serais contenté de prélever quelques échantillons, sélectionnés soit pour leur valeur représentative de l'ensemble soit au contraire en raison de leur singularité. J'aurais pu ainsi commencer cette étude en présentant un spectrogramme, ainsi qu'on nomme une des catégories les mieux pourvues $\mathrm{du}$ corpus, tel le spectrogramme du chant d'une fauvette protonotaire (Prothonotary Warbler) reproduit ci-dessous.

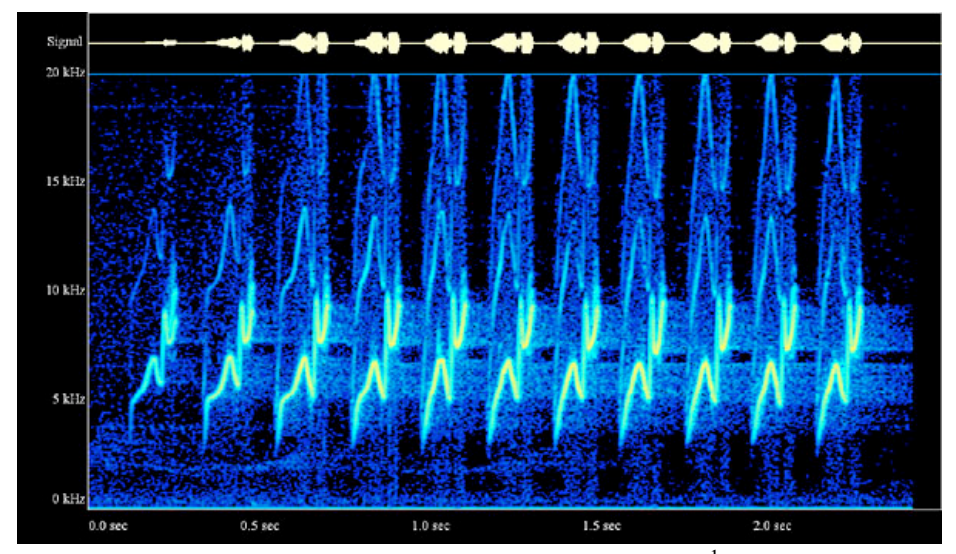

Fig. 1 : Spectogramme du chant d'une fauvette protonotaire ${ }^{1}$

\footnotetext{
${ }^{1}$ « Audio Spectrum of the Song of the Prothonothary Warbler » sur base du Spectrogram Version 8 de Richard Horne, in $\mathrm{http} / /$ :www.visualizationsoftware.com/gram.html.
} 
Cependant, les obstacles théoriques se sont amoncelés au point qu'ils ont rendu problématique la saisie même du corpus. Notamment, et pour faire tenir mes scrupules théoriques sur l'exemple donné, je ne suis pas convaincu que ledit spectrogramme représente une série de sons. À tout le moins, si une représentation peut être maintenue, je ne pense pas qu'elle soit susceptible de saisir la fonction principale du spectrogramme. À quel usage est destiné un spectrogramme ? Dans quel but est-il énoncé ? Voilà une question bien essentielle que la réponse attendue - à représenter des sons —, à laquelle je me fiais d'abord, ne peut que décevoir. À s'en satisfaire, il faudrait reconnaître que ce serait là une représentation très spéciale, car le spectrogramme n'est pas figuratif ; par ailleurs, s'il ne s'agissait véritablement que de représenter des sons, d'autres moyens visuels auraient été disponibles et se seraient avérés certainement plus éloquents ; enfin, dans le cadre d'un texte scientifique, quel intérêt cela aurait-il de donner une représentation de la chose mise à l'examen ? Non, décidément, la notion de représentation est trop déficiente pour qu'on puisse s'en contenter a priori. Or, à ma connaissance, c'est la seule notion dont on dispose jusqu'à présent pour parler de la fonction assignée aux graphiques. Une définition scientifique sera reproduite un peu plus loin. Je me borne pour le moment à ouvrir le dictionnaire Le Robert. Qu'y lit-on ? Graphique, soit adjectif, soit substantif masculin, soit encore substantif féminin (la graphique comme technique de production des graphiques), est toujours défini par la fonction de représentation. Mes soucis se sont ensuite portés sur la polysensorialité. Sans doute le graphique d'un son opère-t-il la rencontre de deux sens, le sens visuel dans lequel il s'énonce et le sens auditif qui est celui de son objet. Pourtant, qualifier de polysensoriel un tel graphique, ce serait en forcer la signification. Le graphique est en réalité strictement visuel. La piste de la polysémioticité se révèle également décevante, car elle est insuffisamment théorisée, et des termes tels que ceux de «transposition », de « transcription » ou de « conversion » y expriment trop souvent le rapport des différents sens ${ }^{2}$. Or je ne crois pas que le graphique convertisse, transcrive ni même transpose l'objet auditif en objet visuel ; et quand bien même on l'admettrait, ce qui

\footnotetext{
${ }^{2}$ On en prend pour témoin cet extrait de la note d'intention pour les journées d'études sur la polysensorialité (Bologne, 24-25 octobre 2003) qui ont servi de scène pour la répétition générale de la présente étude : «Comment donc une image (picturale, photographique, graphique...) nous parle-t-elle, par ses propres moyens spécifiques, non seulement du "voir » (ce qu'on a depuis longtemps étudié), mais aussi du toucher, de l'odorat, ou de l'ouïe ? Loin d'impliquer une analyse iconologique ou pseudo-iconologique de productions visuelles telles les représentations allégoriques des " Cinq sens », cette interrogation viserait la problématique plus générale de la conversion sensorielle. Cette opération de conversion ou de traduction peut être distinguée de la transformation synesthésique - à laquelle elle pourrait évidemment être rapprochée - en ceci qu'il ne s'agit pas, pour le dire simplement, de la façon dont une image peut arriver, par exemple, à nous faire entendre un son, mais bien des opérations par lesquelles elle peut nous le faire voir ».
} 
serait faire bon marché des spécificités du langage graphique, en quoi cela permettrait-il de saisir la co-présence effective des deux sens, en tout cas de certaines de leurs propriétés, dans l'énoncé graphique ? Bref, devant tous ces problèmes, et pour conjurer une étude en diptyque, telles ces mauvaises thèses où la réflexion théorique est réduite en une méthode et l'analyse asservie à une application (ce qui fait perdre à l'une comme à l'autre leur intérêt propre), je renonce à produire cette fois l'analyse du corpus afin de suivre quelques réflexions de type épistémologique à propos des graphiques et de leur description sémiotique. Le spectrogramme présenté d'emblée servira alors de simple prétexte imagé pour une suite qui, je le crains, risque de devenir assez abstraite.

\section{De la représentation à la présentation analytique}

La dernière assurance qui aurait dû me rester, avec cet exemple de spectrogramme, serait d'avoir en main un graphique. Mais cela même s'avère discutable et réclame une argumentation. Sans doute les moyens mis en œuvre pour produire un tel énoncé relèvent-ils sans conteste du système graphique. On peut suivre ici la description très complète qu'en a donnée le géographe Jacques Bertin dans son imposante Sémiologie graphique. Le spectrogramme se présente comme un plan à construction orthogonale dont les axes gradués expriment, en verticalité, des fréquences (exprimées en Hertz) et, en horizontalité, une durée positive (exprimées en secondes). Dans la zone limitée par ces deux axes, des courbes de la forme qu'on appelle « vagues » (waveforms) se superposent et produisent une image graphique, au sens défini par Bertin de « forme significative perceptible dans l'instant minimum de vision » (Bertin 1967 : 142). Il s'agit ici, comme on s'en doute, de considérer le graphique comme relevant d'un système d'expression, pour reprendre la terminologie hjelmslevienne : l'image graphique est une forme sémiotique apte à signifier. Mais qu'en est-il précisément du contenu ? De ce côté, Bertin retient moins le credo sémiotique que celui des logiciens : le contenu est pour lui une constante dont la mise à l'écart est immédiatement effective. N'empêche que c'est par le contenu que Bertin définit la fonction assignée aux graphiques :

LA REPRESENTATION GRAPHIQUE est la transcription, dans le SYSTEME GRAPHIQUE DE SIGNES, d'une pensée, d'une « information » connue par l'intermédiaire d'un système de signes quelconques (Bertin $1967: 8$ ).

Tendue comme définition, la proposition n'est guère acceptable, car elle inclut parmi les définissants (dans le système graphique de signes) ce qu'on cherche précisément à définir. Ne cherchons pas toutefois à en choisir une autre. Qu'il suffise de constater que cette proposition s'applique mal au graphique soumis à notre attention. Car le spectrogramme n'est l'effet d'aucune pensée. Il a été engendré par un système de décodage électronique 
qui n'a jamais eu à «penser» les objets sonores qu'il saisit ${ }^{3}$. Sans doute au terme de pensée Bertin admet-il une paraphrase, par information, qui aurait pu convenir à ce qui reste de l'objet sonore une fois représenté par le spectrogramme. Cependant, assure Bertin un peu plus loin dans son ouvrage, l'information telle qu'il la conçoit, avec ses guillemets, est synonyme de « renseignements à transcrire » et se tient dès lors éloignée de l'acception technique que le terme a reçu en cybernétique. Pas davantage qu'une pensée, on ne saurait dès lors admettre qu'une information dans l'acception ordinaire de « renseignements à transcrire » soit représentée ni qu'elle soit connue, antérieurement, par l'intermédiaire d'un autre système de signes. Quand même les sons émis par un oiseau peuvent être tenus pour sémiotiquement formés, l' « information » exprimée à travers ledit système sonore n'a pas à être connue avant sa saisie spectrographique.

Devant la difficulté que présente le spectrogramme à répondre à la définition avancée par Bertin, quoiqu'en toute apparence il constitue bien un graphique, je ferais volontiers l'hypothèse que les graphiques sont susceptibles de dépasser le cadre d'une sémiotique liée exclusivement aux objets mentaux. Nulle nécessité à ce que l'objet représenté soit une pensée ou une " information ». Bien au contraire, le champ de la représentation graphique mérite d'être ouvert aux objets ou aux phénomènes dans leur plus grande généralité. Toutefois, dans ce cas de figure, ce ne sont pas seulement les termes de pensée et d' "information » qui font difficulté. Ainsi que j'en ai voulu avertir aussitôt le lecteur, c'est l'expression de représentation graphique qui devient elle-même insuffisante. Bertin avait certainement d'excellentes raisons pour s'en contenter, la première étant que le corpus réuni par ses soins répond uniformément à la fonction de représentation. D'un point de vue général, et moins normatif, il faut pourtant reconnaître que les graphiques ne répondent pas toujours à la fonction de représentation d'objets, laquelle est liée au paradigme de la connaissance. Que l'on songe, par exemple, aux usages que les artistes, de Léonard à Panamarenko, font du

\footnotetext{
${ }^{3}$ Ceci, sur quoi je croyais que tout le monde tomberait facilement d'accord, a été vivement contesté. On a fait valoir que les graphiques procèdent de modèles scientifiques et que la représentation des sons, en l'occurrence leur représentation graphique, présuppose nécessairement leur analyse. Comme je ne vois pas en quoi ce que j'avance entre en contradiction avec ces objections, je suppose que le malentendu créé par l'a priori logicien persiste, quand même on commence par avertir qu'on entend bien en être dégagé. Il ne s'agit donc évidemment pas de dire que le graphique n'est en rapport avec aucune sorte de pensée, mais de constater que ces pensées ne constituent pas en l'occurrence l'objet du graphique et qu'on n'a pas à présupposer de systèmes aptes à faire connaître de telles pensées sinon précisément celui qui préside à la production sémiotique des graphiques. Un graphique manifeste ainsi des formes spécifique d'expression et des formes de contenu (peut-être, quant à elles, non spécifiques, mais ce n'est pas rédhibitoire). Un graphique n'est donc pas l'effet d'une pensée ; il en est le moyen. Et par ce moyen ce n'est pas nécessairement une pensée qui est saisie, mais un objet quelconque, par exemple un son.
} 
système d'expression graphique dans leurs œuvres, et qui n'ont pas uniquement la visée représentative dans laquelle Bertin contient les usages graphiques. En fait, c'est la perspective logicienne adoptée par Bertin qu'il faut remettre en cause. Si les objets saisis dans les graphiques ne sont plus seulement des pensées ou des « informations », la question d'un système de contenu spécifiquement associé au système d'expression graphique, ainsi qu'on a l'habitude de l'établir en sémiotique, se pose inévitablement. Dès lors, au lieu de déterminer a priori le contenu graphique par une fonction de représentation, je parlerais volontiers d'une fonction de présentation, qui ne détermine pas ce contenu mais qui se borne à affirmer un niveau énonciatif : le graphique présente quelque chose d'un objet dans des formes d'expression qui lui sont spécifiques et pour des contenus qu'il contribue à déterminer.

Reprenons la réflexion à partir de l'exemple donné. Le chant d'oiseau est un phénomène sonore. En tant que phénomène, il est déjà capable d'autoprésentation, tel un échantillon, et peut être reconnu par les biologistes et les amis de la nature dans des contextes où son identification est recherchée. Ce chant n'est ni une pensée ni une information, et il ne devient signe (de luimême) que dans certains contextes déterminés. Il constitue bien néanmoins, en tant qu'objet, une source antérieure à sa présentation graphique. Il me paraît important de pouvoir pointer cette antériorité, non seulement comme condition de l'énonciation graphique, mais aussi comme effet du faire sémiotique produit par le graphique. Car ce n'est pas une sorte d'équivalence qui s'établit entre la présentation graphique et la présentation sonore du chant, ce que tend au contraire à exprimer le terme de transcription, employé par Bertin dans la définition incriminée, comme ceux de conversion et de traduction qu'on trouve dans les théories sémiotiques de la polysensorialité. Une transcription s'établit entre des textes appartenant à différents systèmes d'écriture, une traduction, entre deux textes appartenant à des systèmes linguistiques distincts, une conversion, entre deux quantités dont les unités diffèrent. À chaque fois, c'est une équivalence qui est établie entre l'objetsource et l'objet-cible, au point que l'objet-source est aboli par l'échange. Une belle traduction, une monnaie de change, la version pour quatuor à cordes d'une composition originellement écrite pour l'orchestre fonctionnent comme, ont les mêmes fonctions que les objets avec lesquels ils ont été échangés, dès lors que leur équivalence sémiotique a été établie. Autrement dit, dans leur principe sémiotique même, une traduction, une conversion et une transcription sont des opérations symétriques, la cible et la source étant commutables. Il existe en outre une possibilité de transitivité entre les objets traduits, convertis ou transcrits. Sans doute une partie de l' « information » risque-t-elle de se perdre dans la chaîne des échanges, mais cette perte reste extérieure au principe sémiotique de la traduction, de la conversion et de la transcription, ainsi qu'aux efforts prodigués pour maintenir une droite observance de ce principe. 
Entre le graphique et son objet, en revanche, il n'y a ni symétrie ni transitivité. Un graphique peut conduire à la réalisation d'un second graphique, mais la relation qui s'établit entre eux n'est pas la même que celle qui s'établit entre chacun d'eux et l'objet qu'ils présentent. La relation n'est donc pas transitive. Et si, d'après le graphique numérique d'un son, il est possible de produire une simulation digitale, cette simulation n'est pas dans le même rapport au son d'origine que l'un et l'autre ne se trouvent vis-à-vis dudit graphique. Là encore, le graphique ne peut se poser en intermédiaire transitif. La relation à l'objet n'est pas davantage symétrique. À partir d'une carte de géographie de la France, quels que soient son degré de précision et le nombre d'informations qu'elle contient, on ne peut reconstituer le pays luimême ; un diagramme retraçant l'évolution du cours d'actions cotées en bourse ne permet pas de comprendre le fonctionnement de la bourse ellemême ; et l'image graphique d'un son n'est pas équivalente à ce dernier, ni ne permet de revenir à lui, non pour des raisons de réalisabilité technique (en cette matière tout porte à croire que la digitalisation vocale parviendra à se rapprocher de la voix humaine au point que leur différence ne sera bientôt plus perceptible) mais parce que le principe même de leur relation sémiotique l'en empêche.

Quelle est cette relation? Entre le graphique et son objet, il faut parler avant tout d'une relation d'analyse. Chez Bertin, tout le poids de l'analyse est rejeté dans une pensée ou dans une information connaissable et même connue préalablement à la production graphique. Encore faudrait-il nous dire comment cette pensée informatrice est connue. Je pense qu'en réalité elle ne se forme, la plupart du temps, que dans la visée de l'énonciation graphique. Lorsque des catégories sont établies, par exemple des catégories de fréquence hertzienne et de durée positive, lorsque des chiffres sont rassemblés selon ces catégories, leur organisation trouve naturellement et de façon privilégiée à s'associer aux unités du système d'expression graphique. La pensée, qui n'est rien d'autre, dans ce cas de figure, que ce que je voudrais appeler plus précisément une analyse, constitue un système de contenu associé dans les graphiques à un système d'expression, et c'est cette association qui définit la fonction spécifique du langage graphique. Il serait vain d'objecter que cette pensée peut être connue au moyen d'énoncés appartenant à d'autres systèmes d'expression. Ce qui importe, c'est qu'aucun système ne s'interpose nécessairement entre le système graphique et son objet. Car c'est bien vis-àvis de cet objet, et non par rapport à d'autres systèmes d'expression, que l'énoncé graphique produit une analyse. La transcription entre le système graphique et un autre système n'est donc pas indispensable et ne rend pas compte de la fonction sémiotique des graphiques, qui est de présenter, d'une manière qui leur est spécifique et que je vais nommer dans un moment, l'analyse d'un objet ou de plusieurs objets.

Mais disons d'abord quelques mots au sujet de l'analyse en général. Une analyse est la description d'un objet en au moins une composante. Pour 
qu'une analyse puisse être produite, il y a une condition apparemment triviale : il faut qu'il y ait quelque chose à analyser. Cela implique néanmoins que ce qui se donne dans l'analyse ne soit pas indifférent à l'analyse ellemême. Non pas que l'analyse ne puisse s'accomplir sur n'importe quel donné ; c'est plutôt qu'à partir du moment où ce donné est donné dans l'analyse, il est déterminé par elle et vis-à-vis d'elle : c'est quelque chose que l'analyse se donne et qu'elle constitue en objet, si tant est que l'objectivité du donné n'a pas été produite préalablement. Le donné est supposé être antérieur et extérieur à l'analyse. Quand même il ne le serait pas effectivement, il demeure quelque chose de cette supposition : l'antériorité et l'extériorité sont des conditions transcendantales à l'apparaître du donné dans l'analyse. Toute analyse suppose ainsi un Dehors et un Passé sans lesquels elle ne peut accéder au donné nécessaire à la constitution des objets analysés.

Le cas du plan d'architecte est à ce titre édifiant. En tant que projection graphique d'un objet encore à construire, il semble se suffire à luimême. En fait, il n'en est rien. S'il ne présuppose un espace réel, ou même fictif (comme c'est le cas avec les constructions utopiques), extérieur à l'espace sémiotique de la présentation graphique, la présomption d'analycité est rendue caduque ; et s'il ne postule la vraisemblance de construction de l'objet architectural projeté, c'est-à-dire s'il n'est pas énoncé à partir d'un savoir empirique, sur base d'analyses précédentes et d'autres objets déjà analysés, l'objet de l'analyse est inséparable du projet d'analyse lui-même et manque de ce fait à l'objectivité empirique qu'on est en droit d'attendre de lui. C'est au demeurant sur cette condition d'antériorité phénoménologique qu'on peut distinguer les usages sémiotiques ordinaires des graphiques de leurs usages rhétoriques. Dans les dessins de Panamarenko, les graphiques et les plans ne présentent des analyses d'objets volants qu'en tant qu'ils sont inséparables des intentions d'analyse, et comme animés par elles ; ce sont dès lors seulement des semblants d'analyses, parce que leur fait défaut, à l'instar des perspectives d'Escher, la possibilité d'objectiver sur le mode d'un donné empirique les constructions d'objets volants ainsi produites.

Voilà pour l'analyse en général. Il faut à présent faire une distinction entre plusieurs types d'analyses. Un premier type peut être qualifié de sémiotique ; c'est, par exemple, le cas des analyses chimiques, qui ont le statut de variables sémiotiques, c'est-à-dire qu'analyse et synthèse, bien que leur présentation soit distincte, ont le même contenu. Un second type est bien connu des sémioticiens, c'est celui des analyses métasémiotiques. Un arbre dans la grammaire générative, un carré sémiotique dans celle de Greimas, présentent des analyses d'énoncé sémiotique. Ils en pointent des propriétés selon un mode considéré comme spécifique à ces énoncés (ils en pointent les formes). Ils décrivent ainsi à un niveau métasémiotique des objets sémiotiques constitués. Il existe enfin un troisième type d'analyses, qui répond des emplois communs du terme (faire l'analyse d'une situation politique ou d'un roman, faire l'analyse radiographique d'un tableau, et 
même faire une analyse, dans la position allongée d'usage) ; je les qualifierai de phénoménologiques. L'analyse sémiotique est transitive et symétrique. L'analyse métasémiotique est transitive mais non symétrique vis-à-vis de la sémiotique objet. Deux analyses métasémiotiques peuvent toutefois être symétriques l'une vis-à-vis de l'autre, puisque, l'une vis-à-vis de l'autre, elles correspondent à des analyses sémiotiques. Quant à l'analyse phénoménologique, elle n'est ni transitive ni symétrique. C'est donc à ce dernier type d'analyse que correspondent en priorité les graphiques.

\section{Une invariante de contenu : l'analyse phénoménologique}

Une analyse graphique n'est pas seulement un problème de transformation syntaxique, comme il en est dans une analyse sémiotique, ou une affaire de représentation, comme c'est le cas avec une analyse métasémiotique. La question que pose une analyse graphique est d'abord celle du passage entre deux ordres phénoménologiques distincts. C'est la question des sens et de leur rapport qui est illustrée par une analyse graphique. Autrement dit, ce n'est pas dans ses formes que le graphique questionne l'objet, mais dans sa substance, dans sa sensorialité ${ }^{4}$. Il présente, dans les formes qui sont spécifiques à son expression, une analyse de la substance sonore. Pour ne pas risquer l'équivoque, j'insiste sur ce point : ce sont bien des formes sémiotiques qui font le caractère analytique du graphique, mais ces formes ne transcrivent pas nécessairement d'autres formes, appartenant à un autre système sémiotique ; ce dont elles permettent

\footnotetext{
${ }^{4} \mathrm{Qu}$ 'on me permette de rappeler que forme et substance sont les termes d'une opposition qui, avec l'opposition de l'expression et du contenu, est à la base de la théorie hjelmslevienne du langage. La forme est définie comme le résultat d'une analyse sémiotique (à propos d'un objet quelconque), tandis que la substance constitue en quelque sorte un « reste »: ce qui n'a pas été analysé par et dans le système sémiotique. Ainsi, par exemple, des sons linguistiques manifestent une forme qui permet leur différenciation avec d'autres sons linguistiques ( $a$ est différent de é ou $o$ ), ce qui ne les empêche pas de connaître une variabilité d'exécution. Cette variabilité est circonscrite dans la mesure où elle ne peut altérer la fonction différenciative des sons (le son $a$ peut être plus ou moins grave ou aigu, court ou long, arrondi, etc., il n'en sera pas moins toujours distinct de é ou $o$ ). Les caractéristiques de ces variations (l'aigu, le grave, l'arrondi,...) constituent autant de caractéristiques de la substance. On considère que la variabilité d'un objet sémiotique est due à sa manifestation nécessaire dans un, voire plusieurs sens (par exemple, on peut aller jusqu'à considérer, dans un modèle très abstrait, que les formes d'une langue se manifestent aussi bien à travers le sens de l'ouïe — par des sons — qu'à travers le sens de la vue - par des caractères d'écriture). Par extension, la substance devient équivalente à la somme des caractéristiques sensorielles, tandis que la forme définit un modèle « abstrait » de l'objet sémiotique. C'est en ce sens qu'il est possible de produire une analyse de la substance : inanalysable dans le système sémiotique, la substance demeure saisissable par d'autres moyens analytiques, métasémiotiques ou phénoménologiques. Ce sont ces moyens qui sont mis en avant dans les énoncés graphiques.
} 
une présentation, c'est d'une substance manifestée dans l'objet et donnée en son ordre phénoménologique propre ${ }^{5}$. Cela, certains graphiques sonores le manifestent de façon exemplaire, c'est-à-dire sans mélange avec d'autres fonctions analytiques. Par exemple, sur un graphique du logiciel Real Player (qui permet l'écoute sur ordinateur d'enregistrements sonores au format MP3), la présentation sonore se réduit à l'expression d'un ordre de succession. La succession, ou plutôt la successivité ${ }^{6}$, exprime bien une propriété sensorielle. Certes, elle se situe à un niveau d'appréhension très abstraite du sens. Mais c'est justement à travers ce caractère abstrait que la substance est sollicitée dans une analyse phénoménologique : la successivité des sons est analysée en des formes spatiales (une ligne graduée et vectorisée ; un curseur), alors qu'elle-même relève de la dimension temporelle. La successivité est donc une forme de contenu : c'est du temps associé dans le langage graphique à de l'espace. Or cette forme de contenu ne nous dit rien des formes d'expression particulières de tel énoncé musical ou de tel texte oral ; elle ne signifie, par analyse de la substance sonore, qu'une propriété du canal auditif.

On m'objectera peut-être que les graphiques de sons composent un corpus particulier dans l'ensemble des graphiques et que je me suis donné des exemples par trop appropriés en alléguant que l'analyse graphique convoque la sensorialité de son objet. Il est vrai que l'étude des graphiques de sons permet de dégager sans ambiguïté l'aspect sensoriel qui y est en jeu. Je voudrais néanmoins soutenir que l'analyse phénoménologique s'applique à tout graphique, quel qu'il soit, et notamment lorsque l'objet analysé dans le graphique est lui-même un objet visuel. La difficulté pour s'en apercevoir réside en trois points que je ne pourrai discuter ici que brièvement. Le premier obstacle est celui-là même qui vient d'être évoqué dans l'exemple du graphique Real Player, à savoir le degré d'abstraction par lequel est appréhendé le sens visuel dans l'analyse phénoménologique. Mais pourrait-il en être autrement ? Tout ce que nous percevons, nous le percevons à travers des formes - et je n'emploie pas ici le terme de forme dans une acception spécialement sémiotique, celles de la phénoménologie husserlienne et de la Gestaltpsychologie sont également bienvenues. Saisir, depuis les formes d'un système, ce qui relève de la substance ne peut se faire qu'à un niveau de généralité très grand, et quand on tente de dégager de tels traits sensoriels, ce ne peut être qu'à un niveau d'abstraction également élevé. Deuxièmement, il

\footnotetext{
${ }^{5}$ Dans la figure 1, les axes expriment bien, il est vrai, des mesures formelles - la hauteur et la durée du chant. Une analyse métasémiotique est donc amorcée. Mais l'image graphique contenue entre ces axes ne se prête guère à cette fonction ; elle la rend peu claire, et comme secondaire.

${ }^{6}$ De quelque manière qu'on voudra nommer cette propriété, il s'agit de la distinguer du syntagmatique, qui est une propriété formelle. Le syntagmatique n'est pas nécessairement affaire de succession, bien que dans la langue elle est manifestée par ce biais, mais bien de combinaison des formes entre elles.
} 
est rare qu'un graphique utilise des moyens seulement graphiques. Le plus souvent, le graphique est au contraire un énoncé syncrétique, où le système d'expression graphique - pour l'essentiel, des lignes, fléchées ou non, et des formes géométriques simples - est associé à d'autres systèmes visuels, comme l'écriture ou le dessin, de sorte que, si les composantes graphiques pointent l'ordre phénoménologique de son objet, celui-ci peut être analysé au sein du même énoncé dans ses formes par des symboles iconiques ou des textes linguistiques. Enfin, troisième difficulté, la distinction que j'ai proposée entre trois types d'analyses est théorique. Mais un énoncé sémiotique est ordinairement capable d'assumer plusieurs fonctions analytiques, par exemple à la fois phénoménologique et métasémiotique. Tel est le cas pour un grand nombre de graphiques, dans le système qui leur est propre (c'est-à-dire sans avoir à considérer les syncrétismes). J'ai pointé chez ceux-ci l'analyse phénoménologique parce qu'elle permet de spécifier leur langage. Il ne faut pas en déduire pour autant que c'est là leur seule fonction analytique, ni même toujours la plus apparente. Si les graphiques sonores constituent un corpus particulier, c'est donc en ceci que les syncrétismes et les fonctions métasémiotiques peuvent y être absents ; ou du moins sont-ils aisément neutralisables.

Pour donner un peu de corps à l'assertion selon laquelle tout graphique présente une analyse phénoménologique de son objet, je propose d'examiner un exemple de graphique qui a pour objet un phénomène visuel.

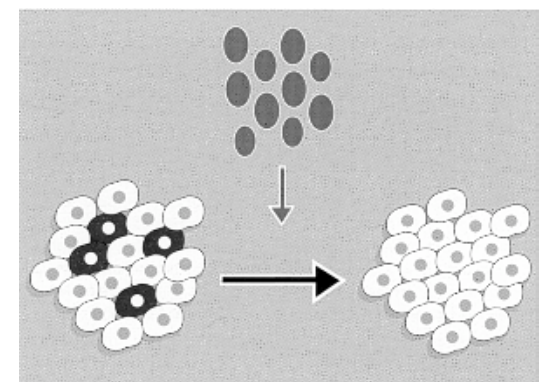

Fig. 2 : Action d'un greffon sur des cellules leucémiques ${ }^{7}$

Ce graphique est composé de deux flèches et de petites formes ovoïdes. La situation énonciative du graphique dans un article de vulgarisation scientifique permet l'identification approximative de ces dernières : ce sont, pour les formes dessinées dans la partie inférieure, des cellules ; elles sont schématisées par deux caractéristiques, l'enveloppe et le noyau. Les ovales parfaits dessinés dans la partie supérieure sont, par comparaison, des éléments corporels indéfinis (la légende indique que ce sont

\footnotetext{
${ }^{7}$ « Leucémies : la recherche en marche », La Lettre du FNRS, 55 (décembre 2003) : 7.
} 
des lymphocytes ${ }^{8}$ ). On laissera de côté les couleurs utilisées dans les dessins comme dans les flèches, puisqu'elles ne sont pas reproduites ici. Qu'y a-t-il de phénoménologique dans l'analyse présentée par ce graphique ? Un moyen pratique de le découvrir est d'isoler par conversion sémiotique l'analyse métasémiotique contenue dans ce graphique. Commençons d'abord par substituer chaque dessin par un symbole. Les symboles utilisés auront, d'une part, à identifier chaque dessin comme un groupe d'éléments, par exemple $\mathrm{A}$ pour le dessin supérieur tel que $A:\left\{a_{1}, a_{2}, a_{3}, \ldots, a_{n}\right\}$, XY pour le dessin inférieur de gauche tel que $X Y:\left\{x_{1}, x_{2}, x_{3}, \ldots, x_{n}, y_{1}, y_{2}, y_{3}, \ldots, y_{n}\right\}, X$ pour le dessin inférieur de droite tel que $X:\left\{x_{1}, x_{2}, x_{3}, \ldots, x_{n}\right\}$; d'autre part, ils auront à marquer la distinction formelle de chaque dessin, deux d'entre eux constituant des groupes homogènes d'éléments $(\mathrm{A}, \mathrm{X})$, le dernier étant hétérogène $(\mathrm{XY})$; enfin, il faudra pouvoir indiquer que l'un des groupes homogènes $(\mathrm{X})$ est en partie identique au groupe hétérogène $(\mathrm{XY})$ tel que $\mathrm{X} \cap \mathrm{XY} \neq 0$. Un premier état de conversion prendrait donc la forme suivante :

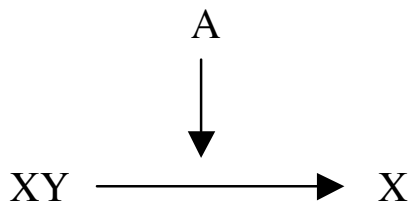

Fig. 3 : Conversion symbolique de Fig. 2

Il est évident qu'un certain nombre de caractéristiques substantielles, relatives à la matérialité et à la corporéité des objets, a été perdu dans la conversion. On n'y retrouve plus, par exemple, la façon dont les cellules sont groupées, à savoir par juxtaposition, ni les différences de grandeurs parmi les éléments corpusculaires, toutes choses qu'en revanche les dessins schématiques signifient de manière évidente. En raison même de la schématicité plus grande des dessins de la partie supérieure, les éléments corpusculaires sont en outre connus pour être peu identifiables visuellement, ce qui constitue, quoique de manière négative, une autre caractéristique de leur substance. Dans une seconde étape, les flèches peuvent être converties en prédicats verbaux, de manière à former la proposition suivante : $\mathrm{XY}$ $<$ devient $>\mathrm{X}<$ en fonction de $>\mathrm{A}$. On peut appeler le langage de conversion adopté ici un langage de formalisation, sans prétendre que ce soit le seul ni le meilleur possible. Il est à tout le moins suffisant pour la conversion visée,

\footnotetext{
${ }^{8}$ Voici la légende dans son intégralité : « La moelle du patient contient des cellules normales (en blanc) et des cellules leucémiques (en rouge [ici en noir]). / Les lymphocytes du greffon (en vert [ici en gris]) vont tuer les cellules leucémiques persistances après la greffe. / Il ne restera que des cellules normales. » (La Lettre du $F N R S, 55: 7)$. Cette légende n'apporte aucune information nécessaire au présent examen.
} 
c'est-à-dire que tout ce que le graphique dit du processus formel à l'œuvre dans l'expérience cellulaire qu'il illustre s'y retrouve'. Dans la conversion des flèches en prédicats verbaux, a-t-on également perdu quelque chose de l'analyse phénoménologique ? Prises en tant qu'éléments autonomes, il est peu probable que les flèches présentent une analyse phénoménologique. Leur emploi récurrent dans les langages formels montre d'ailleurs qu'elles sont aptes à exprimer des contenus sémiotiques et métasémiotiques. Néanmoins, dès lors qu'elles sont associées aux dessins, les flèches permettent de préciser un aspect lié à la substantialité de l'objet qui ne peut pas être pris en charge par les dessins eux-mêmes. Les flèches expriment des types de temporalité distincts, deux formes de durée bien spécifiques. La flèche horizontale marque une durée passive, étale, purement inchoative, et pointe un stade terminal ; c'est la durée même de l'expérience. Et comme il y a quelque chose d'identique et quelque chose de différent entre les objets aux deux bouts de cette flèche, le résultat de l'expérience est compris comme une transformation et, dans ce cas précis, comme une élimination ${ }^{10}$. La flèche verticale, par contre, marque une durée active, imminente ; elle constitue le cœur de l'expérience - en l'occurrence, l'inoculation des lymphocytes. En termes sémiotiques, on dira que les flèches connaissent des valences opposées, la durée horizontale étant extense, la durée verticale, intense. Dans la conversion verbale, ce qui a été perdu, c'est avant tout la netteté de cette opposition. Or l'évidence avec laquelle elle apparaît dans les formes graphiques est une évidence perceptive, liée à la substantialité et à la temporalité de l'expérience.

J'arrête ici le commentaire. Je voudrais néanmoins prévenir trois objections dont la portée théorique va croissant. La première objection consiste à constater que le commentaire à propos de l'analyse phénoméno-

\footnotetext{
${ }^{9} \mathrm{C}$ 'est plutôt la réciproque qui nécessiterait une argumentation. Le prédicat $<$ devient $>$ peut être paraphrasé par « passe d'un état $a$ à un état $b$ »; or la flèche est bien un passage (c'est une ligne) reliant deux objets (symboles ou dessins) dont l'un est antérieur à l'autre, ce qui fait de ceux-ci des états (la flèche est vectorisée). Le prédicat $<$ en fonction de $>$ transpose simplement une subordination de la flèche verticale par rapport à la flèche horizontale, étant donné que celle-ci est pointée par celle-là. Bien entendu, dans l'un et l'autre sens, la perspective de conversion écrase les différences, nombreuses, entre les deux systèmes d'expression.

${ }^{10}$ Un petit test soumis à des personnes non averties l'a confirmé. Présentant d'abord la figure 2 bis, je leur demandais de " transcrire en une phrase la signification du schéma ». Je leur présentais ensuite la figure originale avec la même question. Certaines réponses sont significatives : ceux qui ont écrit que « XY devient X... » à la première question ont employé des formules telles que « les cellules sont transformées... » ou « le passage des petites boules grises fait disparaître... » à la seconde. Je dois toutefois à la probité de reconnaître que le test a manqué le but visé, qui concernait les différences perçues entre les deux schémas, dans de nombreux cas. Mais alors c'est le langage graphique dans son ensemble qui a été interprété de façon surprenante, ce qui pourrait faire l'objet d'une autre étude.
} 
logique conduite par le graphique est fait d'une suite de propositions verbales et qu'il aurait pu ainsi être lui-même intégré à la proposition de conversion. Ce serait pourtant confondre le procédé de conversion avec une analyse seconde, portant sur l'analyse en œuvre dans le graphique. Dans une conversion, il faut prendre soin à la proportionnalité et à la compositionnalité des énoncés convertis. Une variante de l'objection consiste à proposer la légende (citée en note) comme une conversion valable. Mais le graphique ne permet pas d'identifier à coup sûr les objets des dessins (je ne les ai désignés que pour la facilité de l'expression), ce qui ne l'empêche pas d'exprimer à propos de ces objets, indépendamment de leur identification, des caractéristiques substantielles, telles les différences de taille entre composantes et les modalités spécifiques de leurs groupements dans l'espace. Dans une seconde objection, c'est l'exemple même qui est contesté. Est-ce bien là un graphique ? On peut certes en discuter, et affiner les typologies. Je m'en tiens pour ma part à une délimitation maximale : appartient à la sémiotique graphique n'importe quel énoncé visuel qui a une fonction d'analyse et qui ne peut être assigné au langage exclusivement verbal (qu'il soit naturel ou formel) ; les tableaux, les cartes de géographies, les diagrammes et les organigrammes comptent, eux aussi, parmi les représentants les plus typiques relevant de cette sémiotique. L'énoncé graphique ainsi délimité reste perméable aux autres catégories : un énoncé peut être globalement perçu comme relevant de la catégorie des textes, et contenir cependant quelques éléments graphiques; un dessin peut viser globalement une fonction esthétique, et participer, par quelques-uns de ses éléments, à une fonction analytique. Délimiter le domaine d'identification des graphiques et le domaine d'application de leurs éléments spécifiques n'est donc pas suffisant pour les définir. L'exemple examiné est sans doute perçu ordinairement comme ayant une fonction analytique, et par ailleurs son analyse n'est pas exprimée par des mots écrits. Il relève par conséquent, selon la délimitation proposée, de la sémiotique graphique ; en le nommant schéma, on ne désigne, à mon sens, qu'une classe appartenant au plan d'expression de cette sémiotique. La troisième objection porte sur ce qu'il y avait à démontrer. Ne serait-il pas banal, en somme, qu'un schéma signifiât quelque chose de la substance de son objet ? ne serait-ce pas là une façon détournée de dire que le schéma est un signe motivé, partiellement motivé ? La réponse à cette objection pourrait faire l'objet d'un autre article. Au moins puis-je assurer qu'il n'en est rien : l'opposition forme vs substance, d'obédience hjelmslevienne, ne recoupe nullement l'opposition, plutôt saussurienne, signe arbitraire $v s$ signe motivé. Je n'en prends pour garantie que l'énoncé suivant, qui illustre également le problème de délimitation évoqué dans la seconde objection. 


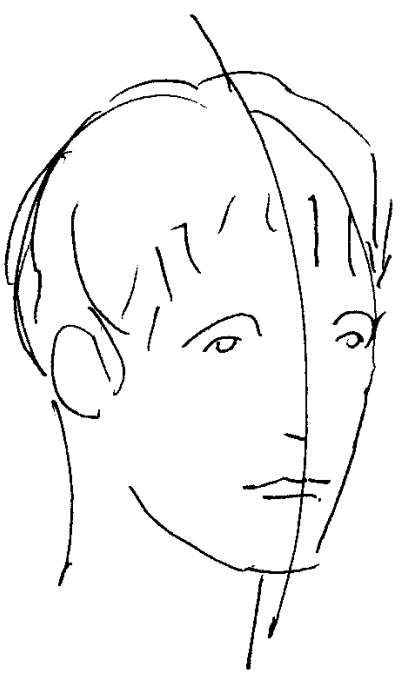

Fig. 4 : Esquisse de tête d'homme ${ }^{11}$

L'énoncé est certainement à ranger dans la catégorie générale des dessins, dont les fonctions ne sont pas a priori analytiques. Plus précisément, on pourrait le désigner sous le terme d'esquisse, ou de dessin préparatoire, ce qui ne fait pas encore de lui un énoncé analytique (il remplirait une fonction de savoir-faire, au lieu qu'un énoncé analytique est plutôt un faire savoir). Ce dessin n'en comporte pas moins un élément devant être considéré comme graphique. C'est la ligne de construction qui se profile sur le visage. Cette ligne relève en effet des moyens utilisés par le système d'expression graphique pour analyser le visuel. D'une part, elle n'a pas un contenu qui soit figuratif, au contraire des autres traits du dessin, ce qui fait précisément d'elle un élément « arbitraire », au sens qu'on donne traditionnellement à ce terme en sémiotique. D'autre part, ce qui est analysé par cette ligne, ce n'est pas l'une des formes de l'objet dessiné, mais bien une propriété du canal visuel, à savoir la possibilité de symétrie (propriété qui ne serait pas pertinente, par exemple, pour le canal auditif). Nous retrouvons donc la trace d'une analyse phénoménologique dans un élément spécifiquement graphique.

Dans les trois cas présentés, qui diffèrent entre eux par leur statut énonciatif et par leur degré de complexité sémiotique, il me semble qu'on a vu que le graphique agit sur l'objet en analysant toujours l'une ou l'autre de ses composantes substantielles : l'ordre manifesté par le curseur sur la ligne n'est compréhensible que par rapport au canal auditif ; les flèches associées aux dessins témoignent de la matérialité de l'expérience dans ses aspects temporalisés ; et la ligne de construction ne signifie la symétrie du visage qu'en vertu des propriétés du canal visuel. En revanche, l'analyse que le

\footnotetext{
${ }^{11}$ (C) Francesco Defourny.
} 
graphique présente de cet objet peut être très secondaires par rapport aux formes sémiotiques que l'objet manifeste (lorsqu'on a affaire à un objet sémiotisable). Par exemple, le graphique d'une chanson, sur Real Player, ne nous dit rien de la durée du morceau, de son volume instrumental, de sa composition, etc. L'analyse effectuée dans le graphique permet de visualiser, elle fait voir quelque chose de son objet. C'est là sa manière spécifique de présenter l'analyse d'un objet, ou de plusieurs objets. Je me propose que nous nous penchions sur ce "faire voir », en laquelle consiste la modalité définitoire des graphiques ${ }^{12}$.

Auparavant, concernant l'analyse phénoménologique présentée par les graphiques, je dois tout de même admettre un cas de figure où elle ne saurait être d'application. C'est celui où l'objet du graphique est effectivement, comme le soutient Bertin, un objet mental et rien d'autre. Par exemple, dans la série de graphiques reproduite ci-dessous, l'appréciation de trois appareils photographiques numériques, sous forme de cotes allant de 1 à 5 , et en fonction de six critères, est rapportée dans une représentation dite « en radar ».

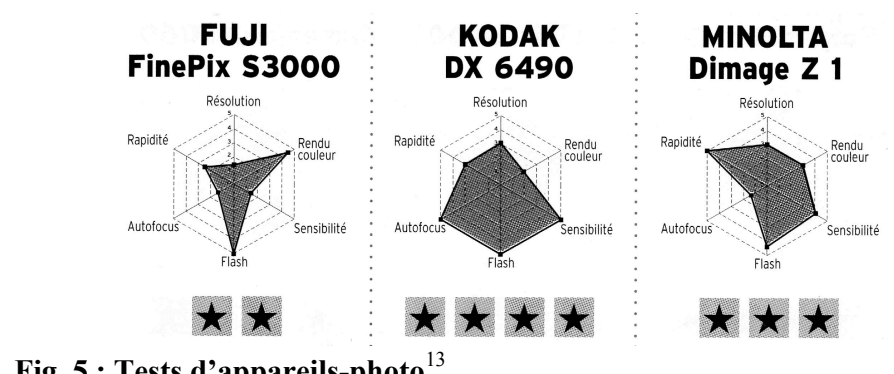

Lorsque l'objet présenté par le graphique est un jugement, on ne saurait déduire de celui-ci une quelconque qualité sensorielle. Sans doute le jugement porte-t-il sur un événement ou un objet qui lui-même se manifeste dans l'un ou l'autre sens, mais le graphique ne présente pas cet objet, du moins pas dans ses moyens propres. Ainsi, dans l'exemple allégué, rapidité, résolution, rendu couleur, sensibilité, flash et autofocus sont des termes qui renvoient aux caractéristiques des appareils photographiques, et dénotent

\footnotetext{
${ }^{12}$ Le concept de « faire voir » m'est apparu dans tout son intérêt à la suite d'une conversation avec Anne-Marie Christin, Yves Jeanneret et Emmanuël Souchier. Qu'ils soient ici remerciés de leurs suggestions. Christin a développé une thèse analogue au bénéfice de l'écriture : "L'écriture ne reproduit pas la parole, elle la rend visible ", écrit-elle (in A-M. Christin dir. 2001 : texte de jaquette). Toutefois, comme on ne saurait considérer que la parole est l'objet — ou, si l'on préfère, le sens - de l'écriture, ce n'est pas son objet que l'écriture rend visible, à la différence de ce qui se passe dans les graphiques. Les deux thèses demeurent distinctes.

${ }^{13}$ Document FNAC 2004 : Sélection Photo numérique, p. 14.
} 
assurément les qualités sensibles de ceux-ci, mais le graphique ne présente pas ces caractéristiques, il se borne à les nommer. À la limite, on pourrait envisager qu'à l'objet conceptuel le graphique invente une substance visuelle. Qu'on ne s'y trompe pas, cependant : cette invention de substance est de pure forme. Dans les graphiques en radar, des formes géométriques, dénotant les qualités du sens visuel, émergent de moyens graphiques (la graduation autour de trois axes, la répartition des six critères aux bouts de ces axes). Ces formes produisent autant d'images graphiques et permettent de ce fait de personnaliser l'évaluation de chaque appareil. Dans leur visualité même, elles n'expriment cependant rien d'autre que la synthèse sémiotique des moyens graphiques.

On ne saurait donc voir d'analyse phénoménologique dans le dernier exemple présenté. On y trouve en revanche une analyse sémiotique (car l'évaluation repose sur des tests objectifs qui ont fait l'objet de présentations graphiques antérieures) et une analyse métasémiotique (notamment à travers les synthèses de ces tests que les formes géométriques permettent de visualiser). Toutefois, dans le souci de préserver à mon hypothèse sur la fonction graphique sa généralité, j'avancerai que l'analyse phénoménologique est simplement suspendue, ou neutralisée, lorsque l'objet ne répond pas aux conditions de cette analyse. De ce fait, l'analyse phénoménologique reste inhérente à la fonction graphique, quand même son application fait défaut. Au demeurant, le corpus graphique que je suis en train de constituer me fait observer que les graphiques d'objets mentaux, sans être rares, ne sont pas aussi massivement représentés que l'ouvrage de Bertin ne le laisse supposer.

\section{Une invariante d'expression : la modalité épivisuelle}

Dire que le graphique «présente une analyse phénoménologique de son objet ", c'est exprimer une fonction sémiotique : on met en corrélation des formes d'expression avec un contenu saisi dans son aspect invariant. Dans cette formule, le terme de présentation, a-t-on prévenu, ne détermine aucune invariante, qu'elle soit d'expression ou de contenu, mais pointe simplement un niveau d'énonciation. On aurait d'ailleurs pu employer un autre terme pour désigner ce niveau, tel le terme de manifestation d'après la terminologie de Hjelmslev. Si je l'ai préféré à d'autres, c'est surtout par opposition au terme employé par Bertin de représentation, lequel infléchit, en la limitant, la fonction graphique. Or, à ce niveau d'énonciation, le terme de présentation doit pouvoir s'appliquer aussi aisément aux formes d'expression qu'aux formes de contenu. On dit indifféremment que des moyens graphiques sont présentés, ou qu'ils sont manifestés, dans un graphique. Mais, ce faisant, a-t-on saisi le mode spécifique de présentation / manifestation des formes graphiques ? Il me semble que non, et que lorsqu'on dit que les moyens graphiques font voir leur objet, on ne dit pas 
seulement qu'ils le présentent ou le manifestent mais on pointe une invariante d'expression.

La sémantique lexicale peut contribuer à nous faire une idée plus précise de celle-ci. Avec représenter, la seule manière de rendre compte du sens du premier graphique présenté en exemple est de dire que le graphique représente un son. Si l'on remplace représenter par présenter, la phrase devient à tout le moins équivoque : le graphique présente un son ou bien signifie que le graphique est un syncrétisme audiovisuel, ou bien, dans le sens qu'on cherche ici à lui donner, utilise une métonymie douteuse. La ratio facilis voudrait que l'on dise plutôt : le graphique présente l'image d'un son. Or, remarquons que faire voir s'emploie aussi facilement avec l'un et l'autre complément : le graphique fait voir un son, le graphique fait voir l'image d'un son; les deux formulations sont également valables. Du reste, faire voir n'a pas l'exclusivité de cette ambivalence. Visualiser connaît un comportement sémantique analogue ; on dira aussi bien le graphique permet de visualiser un son que le graphique permet de visualiser l'image d'un son. Il y a donc au " faire voir », comme expression lexicale et comme concept, une double valeur sémiotique, l'une apparentée à la représentation, l'autre à la présentation analytique.

Quand on admet de dire, selon l'usage, qu'un graphique présente une image, on voit opérer un dédoublement : c'est bien l'image de quelque chose qui est présentée, mais cette image n'est pas autre chose qu'une image graphique. C'est donc en partie lui-même que le graphique présente. Le graphique est à la fois objet actif et objet passif, sujet et prédicat. Il se présente, s'auto-présente dans l'image qu'il donne de son objet ${ }^{14}$. Si nous paraphrasons l'action graphique en distinguant ses deux statuts d'objet, on pourra dire que le graphique présente une image représentant un son, et rien d'autre. On ne pourrait pas dire qu'un graphique représente une image, ni que son image présente un son. Qu'est-ce alors exactement que l'autoprésentation d'une image ? C'est la donner pour visible. C'est affirmer son ordre phénoménologique. Rendre nécessaire, pour ce qui est signifié par l'image, la substance de cette image. Présenter l'image d'un son, ce n'est donc pas seulement rendre ce dernier visuel ; c'est aussi, et avant tout, le rendre visible. Aussi y a-t-il deux régimes de manifestation qui peuvent se

\footnotetext{
${ }^{14}$ Herman Parret me suggère d'employer ici, au lieu du terme d'auto-présentation, celui de présentification, d'après la traduction dans les Idées directrices pour une phénoménologie du terme allemand Vergegenwärtigung. Je ne suis pas en mesure de faire bon usage de sa suggestion. Je la retiens simplement comme un indice de confirmation quant à la nécessité de distinguer représentation (Vorstellung), présentation (Gegenwärtigung) et auto-présentation, que ce dernier soit assimilable ou non à celui de présentification. Parret (2001) propose un commentaire des modes de présences selon la phénoménologie husserlienne. On verra du reste dans la suite de ce texte que je ne me tiens pas moi-même au terme d'auto-présentation, mais que je lui substitue, dans le cadre théorique général des modalités, l'expression modalité épivisuelle.
} 
superposer dans une image : sa visibilité et sa visualité, son apparaître phénoménologique et son état sémiotique. Tout énoncé visuel est évidemment à la fois un phénomène et un objet sémiotique. Mais il appartient à certaines images seulement de s'auto-présenter, comme il appartient peutêtre à d'autres de s'auto-représenter.

J'appelle cette capacité pour une image à s'auto-présenter la modalité épivisuelle de l'image. Le terme est construit sur la modèle de métavisuel. Comme ce dernier, il opère une contraction vis-à-vis d'une expression mieux formée, à savoir épisémiotique visuelle. Et le terme d'épisémiotique indique lui-même la tentative de généraliser un concept utilisé jusqu'à présent par les psychologues et par certains linguistes, concept désigné par le terme d'épilinguistique. Sans m'étendre sur ce sujet, je dirai qu'une modalité épilinguistique est une modalité selon laquelle les locuteurs manifestent dans leur discours verbal des signes qui présentent la discursivité même de leur discours. La modalité épilinguistique se distingue de la modalité métalinguistique en ceci qu'elle s'intègre dans le discours sans qu'il y ait volonté de la part du locuteur de mettre le discours en représentation ou d'énoncer consciemment un second discours sur le premier, ce qui a lieu en revanche avec la modalité métalinguistique. L'épilinguistique n'a d'ailleurs pas nécessairement une visée cognitive. En fait, tous les « messages à fonction phatique », pour reprendre ici la terminologie de Jakobson, s'énoncent selon une modalité épilinguistique. En généralisant le concept d'épilinguistique et en le rebaptisant dès lors du nom d'épisémiotique, les éléments de définition suivants peuvent être avancés : un énoncé épisémiotique signifie quelque chose à propos de son faire; dans ce faire, ce ne sont pas des formes particulières qui sont visées, mais bien la substance même de l'énoncé, dans la généralité de la manifestation ; la signification épisémiotique n'est pas liée à un objectif de connaissance, mais à la présence phénoménologique du sensible, à sa présentation dans l'énoncé même ; l'épisémiotique se donne comme une modalité discursive, sa possibilité ressortit donc au schéma d'une sémiotique dénotative.

Venons-en à la modalité épivisuelle des graphiques. Un graphique ne peut présenter une analyse de son objet qu'en se présentant également soimême. Cette auto-présentation peut être plus ou moins apparente ; elle le sera d'autant plus si son objet appartient à un autre ordre sensoriel. Dans le cas des graphiques de sons, la modalité épivisuelle joue à plein, parce qu'on a alors affaire à deux canaux réputés pour leur complémentarité oppositive, le visuel et l'auditif. Par la modalité épivisuelle, c'est la visibilité du visuel qui est mise en avant ; c'est le visuel dans sa saisie phénoménologique, ou dans sa substance. Cette auto-présentation n'a évidemment pas de visée cognitive. En accentuant la visibilité graphique, on ne cherche pas à parler du graphique ; rien, par conséquent, qui soit redevable d'une modalité métasémiotique. Ce qui est visé par la modalité épivisuelle, c'est une valeur d'usage, un faire voir qui est la fonction même du graphique. 
On se demandera à quelles apparences on reconnaît qu'une modalité épivisuelle régit telle ou telle image. Elle se décèle d'abord par les formes en usage. Les formes graphiques connaissent sans doute des variétés, dues aux possibilités de combinaison des formes entre elles ; en revanche leur marge de variation est assez faible ${ }^{15}$. Elles ne sont jamais très éloignées d'un prototype, de manière à faciliter une reconnaissance automatique. On se rappelle en effet que l'agencement des formes graphiques a souvent pour effet - c'est même, selon Bertin, la règle à suivre — de susciter une seule image perceptuelle. Cette image perceptuelle globale ne pourrait pas être atteinte si les formes graphiques ne se manifestaient pas avec une marge réduite de variations. Mais cette rigidité des manifestations à leurs types suppose aussi un lien fort à la substance visuelle. Que ce soit en matière de supports ou de dispositions énonciatives, les formes graphiques sont conditionnées par leur visibilité. La visibilité est en quelque sorte inhérente $\mathrm{au}$ système. Et puis, la modalité épivisuelle se repère également par les caractéristiques du système de contenu graphique. On l'a vu, le système de contenu graphique est tout entier dédié à l'analyse. Dans cette analyse, les formes de l'objet ne sont pas nécessairement prises en compte ; ce qui est mis en avant, c'est la substance de l'objet. Dès lors, à travers la modalité épivisuelle, d'une part, laquelle relève du système d'expression, et l'analyse phénoménologique, d'autre part, qui ressortit du système de contenu, un choc a lieu entre deux substances. La finalité de cette rencontre n'est pas d'établir une équivalence ou une conversion, d'une substance à l'autre. On pourrait dire au contraire, pour parler comme Eisenstein, que ces substances sont « non indifférentes ». Elles ne se laissent pas échanger mais se soutiennent l'une l'autre : la sonorité, à travers une analyse visuelle qui abstrait ses propriétés telles que la successivité et la compositionnalité ; la visualité, parce que même si le contenu des formes graphiques n'est pas interprétable l'analyse fait apparaître la substance du visuel dans sa condition phénoménologique, c'est-à-dire dans sa visibilité. Remettons-nous le spectrogramme en tête. On peut ne pas savoir ce que "représentent » les formes de vagues, on peut même ignorer que le graphique est un spectrogramme, celui-ci ne manifeste pas moins, à travers des courbes discrétisées, le faire voir d'une analyse portant sur un objet autre que lui, c'est-à-dire sa visibilité même, dans et par le graphique.

\section{Du visible au visuel}

Je voudrais soulever un dernier problème théorique. Il concerne le statut de ce qui est présenté dans le graphique. D’où le sens est-il saisi ?

\footnotetext{
${ }^{15}$ Variété et variation sont des concepts à entendre au sens hjelmslevien : les variétés sont encore identifiables d'un point de vue formel (elles désignent, si l'on veut, les sous-types d'une invariante), tandis que les variations ne le sont pas (elles ne désignent que des différences singulières).
} 
Selon quelle instance ? La modalité épivisuelle présente un faire discursif où l'énonciateur a sa place désignée. L'analyse phénoménologique, semblablement, interroge autant les capacités du sujet percevant que les propriétés de l'objet perçu. De fait, les interrogations sur la substance rendent manifeste la polarité d'un sujet et d'un objet. Dans le domaine visuel, les termes qui expriment le plus nettement cette polarité sont, du côté subjectal, vue, et, du côté objectal, image. Dans le domaine sonore, ce sont oüe et son. Les adjectifs sont en principe neutres par rapport à cette polarisation, de sorte, par exemple, que sens visuel soit à verser du côté du sujet tandis qu'objet visuel le soit évidemment du côté de l'objet. Mais, substantivés, ces adjectifs tendent à se partager les valences de subjectivation et d'objectivation. Ainsi, le visuel tend à appartenir à l'orbe des objets, alors que le visible désigne un rapport subjectal, ce qu'on retrouve également dans le couple adjectival sensoriel / sensible ; c'est pourquoi, dans cet essai, j'ai distingué visualité et visibilité en en faisant un couple phénoméno-sémiotique.

\begin{tabular}{c|c|c|c|c|c} 
subjectal & vue & le visible & oüe & l'audible & le sensible \\
\hline objectal & image & le visuel & son & $\begin{array}{c}\text { l'auditif } \\
\text { ou le sonore }\end{array}$ & le sensoriel
\end{tabular}

Fig. 6 : Polarisation lexicale relative aux sens

C'est en raison de cette dissimilation de la sensorialité qu'une analyse n'est pas symétrique, car les positions de sujet et d'objet n'y sont pas interchangeables : le graphique occupe une position subjectale face à ce qu'il prend pour objet. Si donc le graphique et son objet sont tous deux interpellés dans leur substance, ce n'est pas l'un et l'autre avec la même valence. Quant à son objet, le graphique ne permet nullement de poser la question de l'audible. Au contraire, il aurait tendance à négliger la limite phénoménologique. Ce qui l'intéresse dans la substance de l'objet, ce n'est pas comment elle est perçue par un sujet, mais bien comment elle est conformée par les caractéristiques d'un canal. Par conséquent, non pas l'audible, mais l'auditif. En revanche, devant le sens visuel, la modalité épisémiotique est directement reliée aux capacités de perception du sujet. Ce ne sont pas alors les formes du visuel qui sont auto-présentées dans le graphique (cela, comme je l'ai indiqué, est plutôt du ressort de la modalité métavisuelle) mais bien la substance du visible. Un graphique sonore nous parle de sons mais, en même temps, il porte un regard, moins cognitif que perceptif, sur la vue.

Je m'arrête là. L'examen du corpus des graphiques de sons produirait d'autres interrogations pour la spéculation théorique que ceux qui ont été abordés ici, en amont de cet examen. Il rencontrerait notamment, je l'ai indiqué, la question du syncrétisme, car beaucoup de graphiques sont 
syncrétiques ; en particulier, la plupart des graphiques digitaux sont accompagnés d'un enregistrement sonore. Il appellerait également une réflexion sur les formes métavisuelles, car les graphiques de sons sont souvent accompagnés de légendes et de shifters métalinguistiques. En outre, dans leur forme digitale, ces graphiques sont produits au moyen de logiciels (par exemple, Sound Sculptor) qui présentent les formes graphiques dans leur apparaître sémiotique ; autrement dit ces logiciels fonctionnent comme des métasémiotiques.

En guise d'ouverture à l'étude des graphiques sonores, et aussi pour rassembler dans une analyse les propositions théoriques qui ont été présentées ici, j'esquisserai quelques traits descriptifs à propos du spectrogramme reproduit ci-dessous. On le doit au compositeur de musique électronique Richard D. James, également connu sous le pseudonyme d'Aphex Twin.

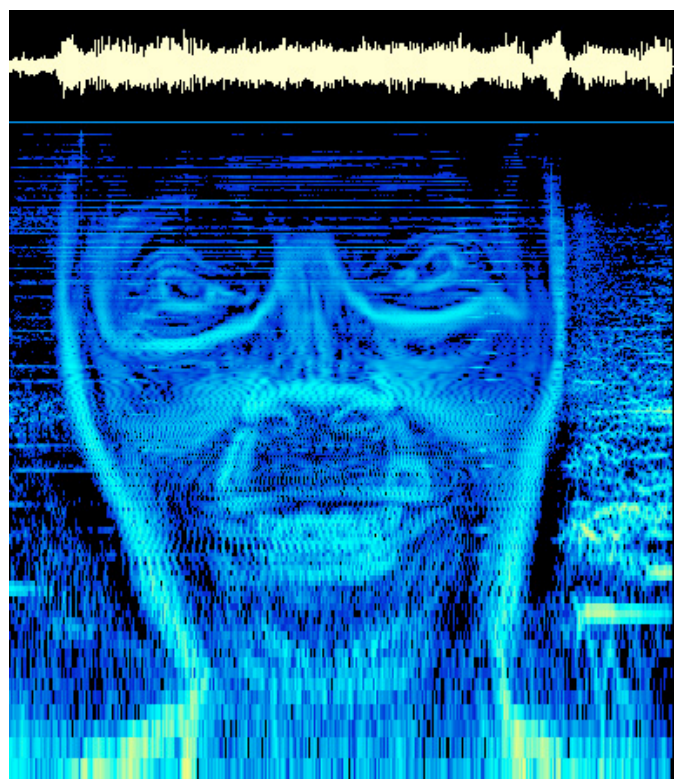

Fig. 7 : Pseudo-spectrogramme ${ }^{16}$

Dès lors que ce graphique présente une analyse, l'évidence est mise sur les propriétés de la substance sonore. On peut le comprendre d'abord par la négative : il serait difficile d'y discerner des formes sémiotiques, étant donné qu'on n'y repère ni régularités ni limites bien tranchées. Ce déficit formel accuse les spécificités de la substance sonore, faite d'intensités, de

\footnotetext{
${ }^{16}$ Aphex Twin, « Equation », in

$\mathrm{http} / /$ :www.visualizationsoftware.com/gram/example 15.html.
} 
hauteurs, de masses, de profondeurs et de seuils. Ces caractéristiques se manifestent dans une temporalité linéaire, ainsi que le présente l'élément graphique supérieur. Il y a ainsi dans ce graphique deux analyses phénoménologiques concomitantes d'une même séquence sonore, et l'une prête à l'autre, par juxtaposition verticale, la caractéristique de la successivité, l'axe du temps étant horizontal. Si cette successivité est si peu apparente dans le graphique inférieur, c'est parce qu'il offre une image tout à fait inattendue du son. Cette image est en effet figurative, et la figure présente une organisation spatiale marquée ${ }^{17}$, contrevenant à la successivité plus ou moins linéaire de la séquence sonore.

On l'aura compris, on se trouve ici devant un graphique sans objet empirique préalable. Son cas est donc analogue à celui d'un plan d'architecte. Et, comme lui, on reconnaît que son analyse se conforme à la substance sonore, exception faite de la figuralité. Il n'aurait pas été envisageable d'utiliser la technique de la ligne claire, ou celle d'un tachisme sans seuils apparents. Ces techniques sont trop éloignées des caractéristiques de la substance sonore, telles qu'elles ont été énumérées plus haut. Mais, par ailleurs, cette analyse n'est pas basée sur la connaissance des formes du son, la figure empêchant même d'y songer, puisqu'aux formes sonores éventuelles le graphique substitue une forme visuelle. Une réalisation sonore, externe au graphique, demeure néanmoins possible. Pour satisfaire à la curiosité du lecteur, je signale en passant que le converter digital produit ainsi, à partir de ce graphique, un son bref et étouffé, sorte de « woup » métallique.

La présence d'une figure marque de façon patente l'activation d'une modalité épivisuelle. Dès lors en effet que la présence de cette figure n'est pas attendue ni justifiée par l'objet du graphique, elle manifeste par surcroît les caractéristiques propres au visuel. Les formes d'expression graphique produisent une forme de contenu spécifique au domaine visuel telle un supplément face à l'objet sonore dont elles sont censées présenter l'analyse. Mais, même en dehors de la figure, on peut reconnaître une interprétation des caractéristiques de la substance sonore au sein du visuel. Les seuils, les densités, les profondeurs sont susceptibles d'une accommodation dans le visuel, et cela non pas en fonction de ses formes mais bien, là encore, en fonction des caractéristiques de sa substance.

J'ai rapproché ce spectrogramme des plans d'architecte. Le rapprochement est évidemment exceptionnel. D'ordinaire, le spectrogramme a un son empirique comme objet préalable à l'analyse. Nous sommes donc en face d'un exemple rhétorique. L'élément qui fait écart par rapport à la norme réside dans l'aspect figural du spectrogramme. Si l'on analyse le mécanisme

\footnotetext{
${ }^{17}$ Dans les termes de Groupe $\mu$, on aurait dû dire que l'image est iconique (et non pas seulement plastique) et construite selon une syntaxe tabulaire (non linéaire) - cf. Groupe $\mu$ 1991. Ces précisions terminologiques sont utiles parce qu'elles rompent avec la théorie de la représentation à laquelle on doit l'opposition, plus commune, du « concret» et de l' « abstrait».
} 
de transformation rhétorique à l'œuvre dans le graphique, on constate à nouveau que c'est la rencontre de deux substances qui y est mise en œuvre. Pour le comprendre, qu'on songe un instant à mettre en parallèle le spectrogramme avec les visages composés de fruits ou de fleurs peints par Arcimboldo. Dans le cas de ces tableaux, un visage est composé d'éléments autres que ceux qu'on attend normalement; et ces éléments autres - des fruits, des fleurs - sont encore figuratifs, sans quoi il n'y aurait pas de transformation rhétorique. Un visage qui serait composé de grosses taches de couleur, ou de petites formes géométriques, relèverait non pas d'une transformation rhétorique mais d'une simple stylisation. Or, dans le cas qui nous occupe, c'est bien le visage qui fait trope, non ses éléments ; et ceux-ci ne sont pas figuratifs. Il n'y a pas stylisation, mais bien émergence d'une figure là où on attendait autre chose. Cette autre chose, ce ne sont pas des formes abstraites qui peuvent y pourvoir par elles-mêmes ; le sens dont on peut doter ces formes serait trop vague et trop général. Il faut au contraire que cette autre chose ait un contenu bien déterminé et qui ne soit cependant pas, en principe, figuratif ; quelque chose, par conséquent, qui échappe à l'opposition de l'abstrait et du figuratif, qui déborde le domaine où cette opposition est pertinente ; bref, il faut que cette chose ne soit pas visuelle. Là où l'on attendait un son, le spectrogramme a présenté une figure.

Et pas n'importe quelle figure. Déployant d'autres manœuvres rhétoriques, à travers cette fois des relations intersémiotiques, le spectrogramme présente la figure d'un spectre. On a l'air de prendre ici le nom du graphique au pied de la lettre : quoi d'autre serait représenté dans un spectrogramme, sinon la figure d'un spectre ? La figure spectrale prête heureusement son concours à mes propositions théoriques : elle n'est que substance, non pas forme, et une substance évanescente, je veux dire qui tantôt disparaît tantôt apparaît. Non moins troublant de coïncidence avec la théorie est le sens étymologique de spectrogramme. Le spectrogramme est l'écriture d'une vision. Ce ne serait pas alors seulement le son qui est rendu figuratif ; c'est le visible lui-même. Auto-présentation du voir dans son apparaître. Le visible s'écrit dans l'apparition d'un spectre au moment où disparaît le son qui constitue sa raison d'être. Les sens ne se représentent pas les uns dans les autres, ni ne se convertissent, semble nous dire ce spectre; ils s'entredévorent. 